\title{
Research on the Impact of Short Selling Mechanism on Investors' Positive Feedback Trading Behavior
}

\author{
Yaoqiong Peng \\ Department of Management, Jinan University, Guangzhou, China \\ Email: MelodyPYQ@163.com
}

Received 19 March 2016; accepted 25 April 2016; published 28 April 2016

Copyright (C) 2016 by author and Scientific Research Publishing Inc. This work is licensed under the Creative Commons Attribution International License (CC BY). http://creativecommons.org/licenses/by/4.0/

\begin{abstract}
With the background of China's Margin Trading pilot, the paper studies the effect of short selling mechanism on investors' positive feedback trading behavior. The research results show that positive feedback trading behavior exists in Chinese stock market, and the launching of the short selling mechanism inhibits effectively the positive feedback trading behavior. Investors can show their pessimism towards the stock price by going short selling, thus the stock price can return quickly to the basic value. At the same time, leverage effect consists in the stock market, namely that investors react more strongly to bad news than to good news. After the implement of margin trading and short selling mechanism, the risk hedging mechanism increases, and investors in the market generally tend to react rationally to good news and bad news.
\end{abstract}

Keywords

Short Selling Mechanism, Behavioral Finance, Positive Feedback Trading Behavior

\section{Introduction}

On March 31, 2010, the A-share market launched the margin trading pilot, so that the credit transactions that were a basic trading mechanism in capital market formally introduced into China. This filled a long time vacancy that lacked of short selling mechanism in securities market. In mature capital market, short selling provides a means of self-correction for the stock price. It is conducive to the discovery of prices, to bring more market transactions and trading strategies. The investors have an opportunity to combine different debt repayment me- 
thods through the margin trading functions, and then derive a variety of trading methods and strategies to achieve different investment objectives.

However, after the stock market crash in June 2015, the Stock Exchanges revised the rules of margin trading, and monitored the abnormal trading account. According to the experts' report, frequent short selling transactions would cause adverse effects when the stock market was down. Then, several securities companies announced the suspension of margin trading business. The securities markets around the world and regions often implement restricted items to limit the short selling transactions when there is a crash in case of a greater impact on the stock price. This paper attempts to carry out an empirical research to contrast the effect of the implementation of short selling on positive feedback trading behavior of investors before and after the implement of the short selling mechanism, hoping to get useful conclusions.

In the study of the mechanism of short sales, first of all, the domestic study mainly concentrated in the analysis of the effect on information disclosure, asset prices and market volatility. Few study revealed the effect of the implementation of margin trading on the behavior of investors. And this is the primary coverage of the paper.

Secondly, the prior domestic research mostly stayed in the theoretical analysis and the simulation stage. This paper divided the empirical data into two parts including before and after the introduction of the short selling mechanism. By constructing the positive feedback trading model test, the empirical results analyzed the effect of margin trading on the investor behavior.

The remainder of this paper proceeds as follows. Section 2 reviews the related literature and develops empirical hypothesis. Section 3 introduces the DSSW model and asymmetric TARCH model, and then describes the data and presents the main analyses of empirical results. Section 4 sets forth our conclusions.

\section{Literature Review and Empirical Hypothesis}

As one of the important link in the chain of financial innovation, Short selling mechanism is generally considered an indispensable part of the stock market. Its appearance not only promotes liquidity of the stock exchange, but also provides a new mechanism for price discovery [1]-[3]. Miller (1977) showed that those investors without holding corresponding private information of the stock cannot reflect the pessimism into the price of the securities when there exists restrictions on short selling and investors have heterogeneous beliefs [4]. Some other studies found that short sellers' manipulation behavior and predatory trading strategies will reduce the price efficiency [5], or lead to excessive reaction of price [6]. Hong and Stein (2003) found that short selling constraints make the price of absorbing information not fast enough, so that the stock price cannot fully reflect the market information (especially negative information) [7]. Bris et al. (2007) found that the negative information is easier to be reflected by the stock price and the stock price crash frequency is lower after that market allows short selling [1], which supports the Hong and Stein (2003) [7]. Liao and Yang (2005) studied the relationship between the short selling and stock prices with the data during August 1998 and February 2004 in Taiwan stock market, and the results showed that there is a long-term stable co-integration relationship between short selling transactions and weighted stock index [8]. Short selling mechanism cannot exacerbate the volatility of stock prices, and to some extent can even restrain the volatility of the stock market. Delong, Shleifer, Summer and Waldmann (1990) showed that the feedback behavior is irrational, which will lead to stock price's significant deviation [9].

Another point of view, on the contrary, market traders need a certain amount of time to absorb new information, that is, the current market price need a certain amount of time to digest all the information and, therefore, does not reflect all information. So the positive feedback trading behavior may be rational [10]. Badrinath and Wahal (2002) showed that when the positive feedback effect leads to price deviation from its fundamental value, the rational arbitrageurs correct this bias with the help of negative feedback strategies, and then the positive feedback traders cannot arbitrage from trading [11]. The exogenous shock is brought about by Margin Trading pilot program. On March 31, 2010, the stock exchange implemented Margin Trading pilot program, which allowed investors to can conduct a credit transaction. These programs significantly relaxed the short-sale constraints on the pilot stocks. As expected, the pilot stocks are increased significantly by short selling activities relative to other stocks.

Building on the above arguments and facts, this paper proposes the empirical hypothesis that margin trading and short selling mechanism implementation can effectively inhibit the investors' positive feedback trading behavior, and the investors' response to good news and bad news tends to be rational. 


\section{Data and Empirical Analysis}

\subsection{Data}

The paper obtains four lists of Index (SSE Composite Index, SHSE-SZSE 300 Index and SSE 180 Index) daily return rate as the research sample. The constituent stocks of SSE Composite index are all listed stocks, including A shares and B shares, reflecting the changing situation of listed stocks of the Shanghai Stock Exchange. SHSESZSE 300 Index selected 300 A-shares in the Shanghai and Shenzhen stock market as a sample compiled index constituent stocks, covering about $60 \%$ of the market capitalization of the Shanghai and Shenzhen stock market, reflecting the overall trend of the two markets. It is "blue chip" in many stock indexes and the concentration camp of many institutional investors' positions. SSE 180 Index selected the most representative 180 stocks as sample stocks, to establish a reflection profile and operation of the Shanghai stock market. The paper used two sample periods which covered Jan1, 2009 to March 30, 2010 and March 31, 2010 to December 31, 2014 as the two contrast sample before and after the implementation of short selling transactions. And this paper made $R_{t}=$ $100\left(\log p_{t}-\log p_{t-1}\right)$.

\subsection{Modeling}

Positive feedback trading strategy is chasing and buying the winners. That is, buying the stock when the trend of stock prices is up, and selling the stock when the trend of stock prices is down. The traders make their investment decisions according to the prior stock price.

Positive feedback trading behavior model is firstly proposed by Shiller (1984) [12]. Sentana and Wadhwani (1992) improved the model on the basis of it [13]. They divided the participants of stock market into rational investors (smart money) — following the value investing, and feedback traders—-taking positive feedback trading strategy. The rational investors take expected return maximization as the investment strategies, and evaluate and trade the stock according to risk return model. The feedback traders' demand is proportional to the stock price change of previous period.

$$
\begin{gathered}
S_{t-1}=\left(E_{t-1}\left(R_{t}\right)-\alpha\right) / \theta \sigma_{t}^{2} \\
P_{t-1}=\rho R_{t-1} .
\end{gathered}
$$

$S_{t-1}$ is the shareholding ratios of rational investors in $t-1$ phase, $R_{t}$ is the index returns in $t-1$ phase, $E_{t-1}$ is the expected value in $t-1$ phase, $\alpha$ is the risk-free return rate, $\sigma_{t}^{2}$ is the conditional variance of return volatility in $t$ phase, $\theta$ is the risk aversion coefficient $(\theta>0), \theta \sigma_{t}^{2}$ is the risk compensation for the stock holders, that is to say, the higher the expected rate of return of investors and the smaller the risk is, the greater the demand of rational investors is; $P_{t-1}$ is the stock price in $t-1$ phase, $\rho$ is the elastic coefficient of demand (positive feedback trading behavior when $\rho>0$, and negative feedback trading when $\rho<0$ ). $\sigma_{t}^{2}$ is measured by $\operatorname{GARCH}(1,1)$ model as Equation (1)

$$
\sigma_{t}^{2}=\alpha_{0}+\alpha_{1} \varepsilon_{t-1}^{2}+\beta \sigma_{t-1}^{2} .
$$

In Equation (1), $\varepsilon_{t-1}$ is the disturbance in $t-1$ phase, $\alpha_{1}+\beta$ represents the persistence of volatility. In the market equilibrium conditions, it satisfies the following equation:

$$
\begin{gathered}
S_{t-1}+P_{t-1}=1 \\
E_{t-1}\left(R_{t}\right)=\alpha+\theta \sigma_{t}^{2}-\theta \rho \sigma_{t}^{2} R_{t-1} .
\end{gathered}
$$

The synchronous reaction of stocks of stock index caused by related market factors will lead to serial correlation of stock index. For example, the price changes of large volume stock will reflect faster than low volume stocks. Assume that $R_{t}=E_{t-1}\left(R_{t}\right)+\varepsilon_{t}$, in order to distinguish the autocorrelations caused by the positive feedback trading, nonsynchronous trading, market failure and other factors, making $\phi_{1}=-\theta \rho$, the model is

$$
R_{t}=\alpha+\theta \sigma_{t}^{2}+\left(\phi_{0}+\phi_{1} \sigma_{t}^{2}\right) R_{t-1}+\varepsilon_{t} .
$$

In Equation (2), when $\phi_{1}$ is negative and statistically significant, it indicates the existence of positive feedback trading behavior. $\phi_{0}$ reflects the impact of market failure or other factors. If $\phi_{0}$ is positive and statistically significant, it means that the non-synchronous trading, market failure and other factors cause autocorrelation. 
At the same time, due to the development of China's stock market is not mature enough, stock price changes will often influenced significantly by policies. In order to reflect the difference of the good news and the bad news reaction of China's stock market better, and illustrate the effects of the introduction of short selling mechanism on investors positive feedback trading behavior better, the paper cites the asymmetric TARCH proposed by Zakoian (1994) [14] and Glosten et al. (1993) [15]:

$$
\sigma_{t}^{2}=\omega_{0}+\omega \mu_{t-1}^{2}+\gamma S_{t-1} \mu_{t-1}^{2}+\lambda \sigma_{t-1}^{2} .
$$

In Equation (3), the $S_{t-1}$ is a dummy variable. When $\mu_{t-1}<0, S_{t-1}=1$, or $S_{t-1}=0$. As long as the $\gamma \neq 0$, there exists asymmetric effect. In the formula, $\gamma S_{t-1} \mu_{t-1}^{2}$ in the conditional variance equation is called asymmetric effect, or TARCH. The conditional variance equation shows that the conditional variance depends on the residual square $\mu_{t-1}^{2}$ and conditional variance $\sigma_{t-1}^{2}$. Good news $\left(\mu_{t-1}>0\right)$ and bad news $\left(\mu_{t-1}<0\right)$ have different effects on the conditional variance: the good news has $\omega$ times impact. But there is $(\omega+\gamma)$ times impact in the good news. This is because when $\mu_{t-1}<0, S_{t-1}=1$, which produces $(\omega+\gamma)$ times impact. If $\gamma>0$, it indicates the existence of leverage effect, and the main effect of asymmetric effects increase the fluctuations. If the $\gamma<0$, the asymmetric effects decrease the volatility.

\subsection{Descriptive Statistics}

Table 1 is the statistical characteristics of the daily return rate of four indexes. The distributions of two time periods of the SSE Composite Index, SHSE-SZSE 300 Index and SSE 180 Index have different degrees to skew to the left or right side. The kurtosis values are greater than the kurtosis value of normal distribution, explaining that the protruding degree of the distribution of series is greater than the normal distribution. The J-B statistics show that the return series refused to obey the normal distribution assumption.

\subsection{Empirical Results}

Table 2 reports results from estimating Equation (1) and Equation (2). According to the estimation results of the above test, $\mathrm{Z}$ statistic of parameter of variance equation $\alpha_{1}$ and $\beta$ are significant. The estimated value $\phi_{1}$ of SSE Composite Index, SHSE-SZSE 300 Index and SSE 180 Index are significantly negative before the short selling, but not all significant and turn from negative to positive after the implementation of short selling, which represents investors positive feedback trading behavior is suppressed after that the implementation of short selling mechanism. The estimated value $\phi_{1}$ of SSE Composite Index is significantly negative before and significantly positive after the implementation of short selling. And the estimated value $\phi_{1}$ of SHSE-SZSE 300 Index is significantly negative before and after the implementation of short selling, so as the SSE 180 Index. To explain it, the sample stocks of SSE Composite Index are not all the margin stocks, so the effect of margin trading and short selling on investors' positive feedback trading behavior is not significant enough. However, almost all of SHSE-SZSE 300 Index and SSE 180 Index Stocks are margin targets, so as shown by the empirical results, the implementation of short selling mechanism effectively inhibit investors' positive feedback trading behavior.

Table 3 reports results from estimating Equation (3). The estimation results of asymmetric impact model show that $\gamma$ is all greater than 0 in Group 1, indicating that the investors' reaction to bad news is more strongly than good news. Preliminary analysis suggests that this mainly because implementation of the short selling mechanism was restrained. Once the bad news spreads, investors can only benefit from the buying mechanism, resulting that the small and medium-sized investors can only avoid further losses by selling. So the impact of bad news is greater than the impact of good news.

The value of $\omega$ reflects the impact of good news and the value of $\omega+\gamma$ reflects the impact of bad news. After the implementation of short selling mechanism, all of the value of $\omega+\gamma$ decreases. This indicates that after the introduction of the short selling mechanism, the investors generally react more rationally to the emergence of bad news on the stock market, thus restrains the positive feedback trading behavior in the stock market.

\section{Conclusions}

By combining with the DSSW model and asymmetric TARCH model, this paper makes an empirical comparative analysis of the data before and after the implementation of the short selling mechanism in China. Through the theoretical deduction and empirical analysis, it can be concluded as following. 
Table 1. The statistical characteristics of the daily return rate.

\begin{tabular}{cccc}
\hline \multirow{2}{*}{ Items } & \multicolumn{3}{c}{ Daily Return Rate } \\
\cline { 2 - 4 } & SSE Composite Index & SHSE-SZSE 300 Index & SSE 180 Index \\
\hline Mean & 0.000620 & 0.000760 & 0.000724 \\
Standard Deviation & 0.017892 & 0.019199 & 0.019266 \\
Skewness & -0.417967 & -0.383340 & -0.352948 \\
Kurtosis & 6.418799 & 5.771163 & 5.885088 \\
J-B Statistics & 1253.665 & 836.7033 & 892.8622 \\
p-Value & 0.000000 & 0.000000 & 0.000000 \\
\hline
\end{tabular}

Table 2. The regression results of DSSW Model.

\begin{tabular}{ccccccc}
\hline \multirow{2}{*}{ Items } & \multicolumn{2}{c}{ SSE Composite Index } & \multicolumn{2}{c}{ SHSE-SZSE 300 Index } & \multicolumn{2}{c}{ SSE 180 Index } \\
\cline { 2 - 7 } & Group 1 & Group 2 & Group 1 & Group 2 & Group 1 & Group 2 \\
\hline \multirow{2}{*}{$\alpha$} & 0.002351 & 0.000304 & 0.002371 & 0.000215 & 0.002514 & 0.000171 \\
& $(1.804762)$ & $(0.522399)$ & $(1.683619)$ & $(0.304902)$ & $(1.799939)$ & $(0.243792)$ \\
$\theta$ & -2.791913 & -0.400187 & -2.010144 & -0.003922 & -2.349002 & 0.116467 \\
& $(-1.197713)$ & $(-0.202152)$ & $(-0.890665)$ & $(-0.001808)$ & $(-1.059158)$ & $(0.05504)$ \\
$\phi_{0}$ & 0.153788 & 0.007836 & 0.178638 & 0.004868 & 0.164331 & 0.010385 \\
& $(2.059767)^{* * *}$ & $(0.186805)$ & $(2.304188)^{* *}$ & $(0.11247)$ & $(2.157563)^{* *}$ & $(0.240985)$ \\
$\phi_{1}$ & -208.1230 & 142.8628 & -190.8845 & 107.5139 & -190.0514 & 73.09347 \\
& $(-2.240692)^{* *}$ & $(2.103394)^{*}$ & $(-2.154074)^{* * *}$ & $(1.537544)$ & $(-2.208615)^{* *}$ & $(1.089568)$ \\
$\alpha_{0}$ & 0.00000341 & 0.00000283 & 0.0000018 & 0.00000399 & 0.0000035 & 0.0000042 \\
& $(2.091966)^{* * *}$ & $(4.895807)^{* * *}$ & $(2.18718)^{* * *}$ & $(4.600879)^{* * *}$ & $(1.956839)^{*}$ & $(4.815853)^{* * * *}$ \\
$\alpha_{1}$ & 0.072325 & 0.052258 & 0.011097 & 0.050736 & 0.066624 & 0.053207 \\
& $(6.858148)^{* * *}$ & $(7.895717)^{* * *}$ & $(6.221614)^{* * *}$ & $(6.913897)^{* * *}$ & $(6.120176)^{* * *}$ & $(7.386013)^{* * *}$ \\
$\beta$ & 0.923433 & 0.93367 & 0.010971 & 0.933314 & 0.929195 & 0.9303 \\
& $(90.70697)^{* * * *}$ & $(125.0082)^{* * * *}$ & $(84.39165)^{* * *}$ & $(109.3732)^{* * * *}$ & $(85.87972)^{* * * *}$ & $(113.5822)^{* * * *}$ \\
\hline
\end{tabular}

$*, * *$ and ${ }^{* * *}$ denote the significance level of $10 \%, 5 \%$ and $1 \%$.

Table 3. The regression results of asymmetric TARCH model.

\begin{tabular}{|c|c|c|c|c|c|c|}
\hline \multirow{2}{*}{ Items } & \multicolumn{2}{|c|}{ SSE Composite Index } & \multicolumn{2}{|c|}{ SHSE-SZSE 300 Index } & \multicolumn{2}{|c|}{ SSE 180 Index } \\
\hline & Group 1 & Group 2 & Group 1 & Group 2 & Group 1 & Group 2 \\
\hline \multirow{2}{*}{$\omega_{0}$} & 0.083672 & 0.0000002 & 0.00000519 & 0.00000279 & 0.00000563 & 0.00000417 \\
\hline & $(2.739673)^{* * * *}$ & $(2.705461)^{* * *}$ & $(2.479394)^{* * * *}$ & $(4.80066)^{* * *}$ & $(2.523195)^{* * *}$ & $(4.660592)^{* * *}$ \\
\hline \multirow[b]{2}{*}{$\omega$} & 0.049443 & 0.05344 & 0.062871 & 0.054286 & 0.060544 & 0.047011 \\
\hline & $(2.920160)^{* * * *}$ & $(5.557459)^{* * *}$ & $(4.098672)^{* * * *}$ & $(6.566535)^{* * * *}$ & $(4.192624)^{* * * *}$ & $(5.499854)^{* * * *}$ \\
\hline \multirow{2}{*}{$\gamma$} & 0.054877 & -0.008817 & 0.027241 & -0.0045 & 0.023512 & 0.008573 \\
\hline & $(2.804220)^{* * *}$ & $(-1.017516)$ & (1.627104) & $(-0.511141)$ & (1.460334) & $(0.951414)$ \\
\hline \multirow{2}{*}{$\lambda$} & 0.907604 & 0.940361 & 0.915255 & 0.934117 & 0.919213 & 0.932044 \\
\hline & $(65.78585)^{* * *}$ & -82.01937 & $(82.4307)^{* * *}$ & $(123.1511)^{* * *}$ & $(77.87705)^{* * *}$ & $(108.2906)^{* * *}$ \\
\hline$\omega+\gamma$ & 0.10433 & 0.040635 & 0.090112 & 0.049786 & 0.084056 & 0.055584 \\
\hline
\end{tabular}

*, *** and ${ }^{* * *}$ denote the significance level of $10 \%, 5 \%$ and $1 \%$.

First, the stock market exists positive feedback trading behavior, which supports the study of $\mathrm{Li}$ and $\mathrm{Gu}$ (2007) [16]. Positive feedback trading behavior is a common phenomenon in the global stock market. Second, 
the implement of short selling mechanism can restrain the positive feedback trading behavior of the investors. The function of short selling mechanism to make the stock price return to the base value has been reflected. But there still exists such chasing behavior. However, after the implementation of the sale space mechanism, investors can express their pessimism towards the stock price by short selling, which makes the price return to the basic value quickly. Third, there exists certain leverage effect in the stock market. The investors respond more strongly to bad news than to good news. When the stock market returns decline, investors will further exacerbate the market panic, and then the volatility of the market shows the characteristic of non-symmetry. Fourth, after the introduction of short selling mechanism, the stock market increases the risk hedging mechanism. Therefore, the investors' reaction towards the good news and the bad news generally tends to be rational.

The results of the study showed that the implementation of the mechanism of short selling can restrain investors' positive feedback strategies. This attributes to the role of risk hedge of short selling mechanism, which helps the price of stocks reflect the true value and strengthen the overall investor rational investment. The implementation of short selling mechanism inhibits the overall positive feedback trading behavior of stock market. According to the previous theoretical analysis, most investors in the stock market can express their own pessimism by short selling, which is one of the reasons of widely implementation of short selling mechanism.

At present, according to the situation of short selling mechanism in China, we can find that the amount of short selling is too small compared with the overseas capital market, and the number and the type of the targets are limited, which mean that the development of China's short selling mechanism is not mature enough. At the same time, the access threshold of current short selling trading is too high for the most of medium and small investors. Above all, the beneficial impact of the short selling short mechanism on the stock market is still relatively limited. Based on the above analysis, this paper puts forward the following suggestions. First of all, securities policy makers should strengthen market supervision and improve the mechanism of information disclosure consciously when establish regulatory measures. Secondly, CSRC should improve the long-term construction of short selling mechanism and reform the bonus system which perfecting the profit model. Finally, all of investors should strengthen the awareness of risk of securities investment, especially the small and medium investors, and promote the rational investment.

\section{References}

[1] Bris, A., Goetzmann, W.N. and Zhu, N. (2007) Efficiency and the Bear: Short Sales and Markets around the World. Journal of Finance, 62, 1029-1079. http://dx.doi.org/10.1111/j.1540-6261.2007.01230.x

[2] Boehmer, E., Jones, C.M. and Zhang, X. (2008) Which Shorts Are Informed? Journal of Finance, 63, 491-527. http://dx.doi.org/10.1111/j.1540-6261.2008.01324.x

[3] Boehmer, E. and Wu, J.J. (2013) Short Selling and the Price Discovery Process. Review of Financial Studies, 26, 287-322. http://dx.doi.org/10.1093/rfs/hhs097

[4] Miller, E.M. (1977) Risk, Uncertainty, and Divergence of Opinion. Journal of Finance, 32, 1151-1168. http://dx.doi.org/10.1111/j.1540-6261.1977.tb03317.x

[5] Goldstein, I. and Guembel, A. (2008) Manipulation and the Allocational Role of Prices. The Review of Economic Studies, 75, 133-164. http://dx.doi.org/10.1111/j.1467-937X.2007.00467.x

[6] Brunnermeier, M.K. and Pedersen, L.H. (2005) Predatory Trading. Journal of Finance, 60, 1825-1863. http://dx.doi.org/10.1111/j.1540-6261.2005.00781.x

[7] Hong, H. and Stein, J.C. (2003) Differences of Opinion, Short-Sales Constraints, and Market Crashes. Review of Financial Studies, 16, 487-525. http://dx.doi.org/10.1093/rfs/hhg006

[8] Liao, S.G. and Yang, C.J. (2005) The Short Selling Mechanism, Volatility and Liquidity: An Empirical Study Based on Hongkong Stock Market. Management World, 12, 6-13, 171.

[9] De Long, J.B., Shleifer, A., Summers, L.H., et al. (1990) Positive Feedback Investment Strategies and Destabilizing Rational Speculation. Journal of Finance, 45, 379-395. http://dx.doi.org/10.1111/j.1540-6261.1990.tb03695.x

[10] Shleifer, A., Lakonishok, J. and Vishny, R.W. (1992) The Impact of Institutional Trading on Stock Price. Journal of Financial Economics, 32, 23-43. http://dx.doi.org/10.1016/0304-405X(92)90023-Q

[11] Badrinath, S.G. and Sunil, W. (2002) Momentum Trading by Institutions. Journal of Finance, 57, 2449-2478. http://dx.doi.org/10.1111/1540-6261.00502

[12] Shiller, R.J. (1984) Stock Prices and Social Dynamics. Brookings Papers on Economic Activity, 1984, 457-498. http://dx.doi.org/10.2307/2534436 
[13] Sentana, E. and Wadhwani, S.B. (1992) Feedback Traders and Stock Return Autocorrelations: Evidence from a Century of Daily Data. Economic Journal, 102, 415-425. http://dx.doi.org/10.2307/2234525

[14] Zakoian, J.M. (1994) Threshold Heteroskedastic Models. Journal of Economic Dynamics \& Control, 18, 931-955. http://dx.doi.org/10.1016/0165-1889(94)90039-6

[15] Glosten, L.R., Jaganathan, R. and Runkle, D.E. (1993) On the Relation between the Expected Value and the Volatility of the Nominal Excess Return on Stocks. Journal of Finance, 48, 1779-1801. http://dx.doi.org/10.1111/j.1540-6261.1993.tb05128.x

[16] Li, S.P. and Gu, G.C. (2007) An Empirical Study on the Positive Feedback Trading of Chinese Stock Market. Systems Engineering, 25, 111-115. 